\title{
Expectant management in postlaparoscopic distal emphysema
}

\author{
Paweł Milart, Piotr Czuczwar, Ewa Woźniakowska, Piotr Szkodziak, Tomasz Paszkowski \\ $3^{\text {rd }}$ Chair and Department of Gynecology Medical University in Lublin, Poland
}

We present a case of subcutaneous emphysema as a complication during laparoscopic assisted supracervical hysterectomy (LASH).

Symptomatic subcutaneous emphysema occurs in $0.43-2.34 \%$ of laparoscopic abdominal procedures. Risk factors of this complication include inadequate insufflator settings (high gas flow and high gas pressure setting), intra-abdominal pressure $>15 \mathrm{~mm} \mathrm{Hg}$, improper placement of the trocars, repeated attempts to generate pneumoperitoneum, torqueing of the trocar, procedures lasting $>3.5$ hours.

A 50-years-old patient, weight $62 \mathrm{~kg}$, height $164 \mathrm{~cm}, \mathrm{BMI} 23.05$, hemoglobin normal, was admitted to the hospital because of uterine fibroids. She reported abnormal uterine bleeding and lower abdominal pain. . Ultrasound examination showed the uterus of $8.5 \times 8.2 \times 7.6 \mathrm{~cm}$ with numerous fibroids. The patient was qualified for LASH with bilateral salpingectomy. The surgery was performed in Trendellenburg position. The intraabdominal $\mathrm{CO} 2$ pressure was set to $12 \mathrm{~mm} \mathrm{Hg}$ with the gas flow of $3 \mathrm{~L} / \mathrm{min}$. Three trocars were placed typically, without any difficulties.

One hour after the beginning of the operation a subcutaneous emphysema was observed, which covered the left side of the face, left side of the chest and left upper limb. The occurrence of the emphysema was preceded by intense maneuvers of the left trocar, due to difficulties during the amputation of the corpus of the uterus with an electric loop. Crepitus was palpable in the clavicular regions on both sides of the chest, the left side of the neck, the left cheek and the left temporal region. Both left eyelids were swollen (Fig. 1). On postoperative day one, the crepitus was still present on both clavicular regions of the chest and on the left side of the neck. It was not palpable on the left temporal region, the patient had less difficulty with opening of the left eye; however, she still complained about swollen eyelids (Fig. 2). On postoperative day two, the crepitation was palpable only on the right clavicular side of the chest, without any subjective symptoms. The eyelids appeared normal (Fig. 3).

Apart from the emphysema there were no other complications. The patient was discharged from hospital on postoperative day three. During the further postoperative period, no symptoms typical for subcutaneous emphysema were observed. In our case, the most likely cause of subcutaneous emphysema were extensive maneuvers of the trocar, that caused subcutaneous

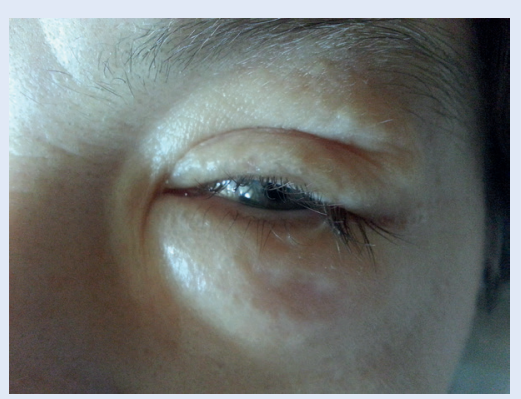

Figure 1. Two hours after the operation. Massive emphysema of both eyelids

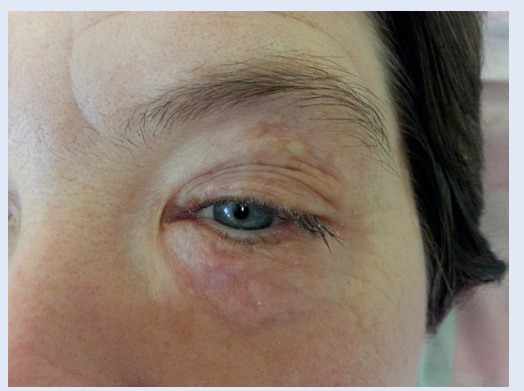

Figure 2. Postoperative day 1. Emphysema visible in lower eyelid

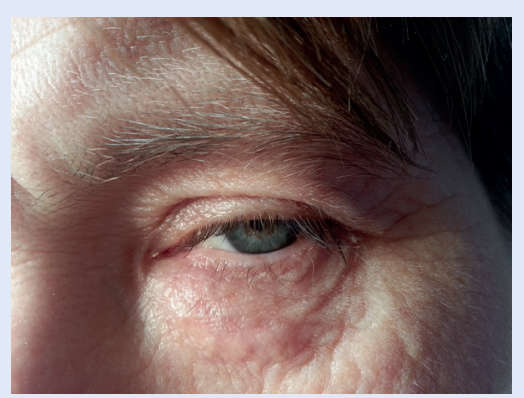

Figure 3. Postoperative day 2. Normal eyelids 
$\mathrm{CO}_{2}$ inflation. To decrease the risk of subcutaneous emphysema during laparoscopy, attention should be given especially to proper insufflator settings and avoidance of torqueing of trocars. In cases where laparoscopy is complicated with subcutaneous emphysema with light/moderate symptoms, expectant management should be recommended and the symptoms should resolve spontaneously. 